\title{
Nonlinear Acceleration Controller for Exo-Atmospheric and Endo-Atmospheric Interceptors with TVC
}

\author{
Chang-Hun Lee ${ }^{1}$, Byung-Eul Jun ${ }^{2}$, Hyo-Sang Shin ${ }^{3}$, and Antonios Tsourdos ${ }^{4}$
}

\begin{abstract}
In this paper, we propose a nonlinear acceleration controller that can be used for both the endo- and exoatmospheric interceptors with thrust vector control (TVC) without changing the control configuration. The acceleration perpendicular to the velocity vector is selected as the output to be controlled. Then apply the feedback linearization and the specific form of the desired error dynamics to create the resulting controller which is given by the well-known three loop control structure with parameter-varying control gains. According to changes in altitude operating conditions, the proposed controller can adaptively allocate the aerodynamic force and the thrust to produce the required normal acceleration. Also, we can have confidence in the reliability of the proposed controller because it is given by a similar form of the well-known three loop controller. Numerical simulations are performed to show the validity of the proposed method.
\end{abstract}

\section{INTRODUCTION}

In recent years, there have been many applications using thrust vector control (TVC). For example, TVC has been used to control the attitude of the launch vehicle system. According to reference [1], some fighters adopted TVC to achieve fast manoeuvrability. Additionally, TVC has been used in spacecraft [2]. Also, in interceptor systems, TVC is widely applied as a means of controlling of interceptors during the midcourse phase [3] as well as the terminal homing phase.

In recent interceptor applications, the interceptor guidance system generates the steering command of the flight path angle (FPA) so that the interceptor reaches a target position, and the control system generates the control commands to track the FPA of the interceptor to the desired value. For this purpose, the existing control strategy of the interceptor equipped with TVC is the attitude control. The control system rotates the attitude of the interceptor so that the interceptor is aligned with the desired direction (i.e., the desired FPA direction). When the attitude angle reaches a target value, the altitude angle is also aligned with the FPA within a short time due to the aerodynamic force. Therefore, the desired FPA can be achieved by the attitude control.

However, as high altitude area defence is generally required in recent years, an interceptor equipped with a TVC is required to have the ability to fly in both endo-atmosphere

\footnotetext{
${ }^{1} \mathrm{C}$. H. Lee is with the school of aerospace, transportation, and manufacturing, Cranfield University, Cranfield, Bedford, MK43 0AL, UK lckdgns@gmail. com

${ }^{2}$ B. E. Jun is Agency for Defense Development, Daejeon, 34168 Korea

${ }^{3}$ H. S. Shin is with the school of aerospace, transportation, and manufacturing, Cranfield University, Cranfield, Bedford, MK43 0AL, UK

${ }^{4} \mathrm{~A}$. Tsourdos is with the school of aerospace, transportation, and manufacturing, Cranfield University, Cranfield, Bedford, MK43 0AL, UK
}

and exo-atmosphere. Accordingly, in the exo-atmosphere, this control strategy is not suitable for achieving the desired FPA because the attitude angle takes some time to converge to the FPA due to the absence of aerodynamics. Recently, to overcome this difficulty, it has been widely considered to use the attitude control in the endo-atmosphere only, and to use the normal acceleration (acceleration normal to velocity vector) control by changing the configuration of the controller in the exo-atmosphere. However, there is ambiguity as to when to change the configuration of the controller in this approach. Additionally, the attitude control in the endo-atmosphere is not a way to effectively use infinite aerodynamic forces to achieve the control objective.

In order to solve the above problems, in this paper, we propose a nonlinear autopilot for the interceptor which can be applicable to both endo- and exo-atmosphere without changing the control configuration. In both flight environments, we select the normal acceleration as the output to be controlled. The feedback linearization technique is then applied along with the specific desired error dynamics [4-7] to design the proposed controller. To investigate the performance of the proposed method, a simulation study using a nonlinear interceptor model [8] is performed. Compared to the attitude control, the proposed controller can effectively use infinite aerodynamic resources in the endo-atmosphere. Also, under the proposed controller, the aerodynamic force and the thrust are automatically allocated to produce the normal acceleration required according to the effectiveness of the aerodynamic force and the thrust. Finally, the result controller is given by a similar form of the well-known threeloop controller [4-7] with parameter-varying control gains. Therefore, the physical meaning of the proposed controller is clearly presented. Also, we can have confidence in the reliability of the proposed controller when implementing it to a real system.

This paper is structured as follows. The problem formulation is explained in Section II. The design and discussion of the proposed controller is given in Section III. The simulation study is performed in Section IV. Finally, we conclude our study in Section V.

\section{PROBLEM FORMULATION}

\section{A. Derivation of Dynamics for Interceptors}

This section describes the flight dynamics of the interceptor used in this study. We consider an interceptor with TVC shown in Fig. 1. When designing autopilot, gravity can be ignored because an acceleration command is generated to compensate gravity in the guidance loop. It also assumes 
that the roll channel can be stabilized quickly. Under the assumption, the lateral motion of the interceptor can be split into two perpendicular channels (pitch motion and yaw motion). If the configuration of the interceptor is symmetric, the pitch and yaw motions are the same. Therefore, only pitch motion is considered in this study. In Fig. 1, the coordinate

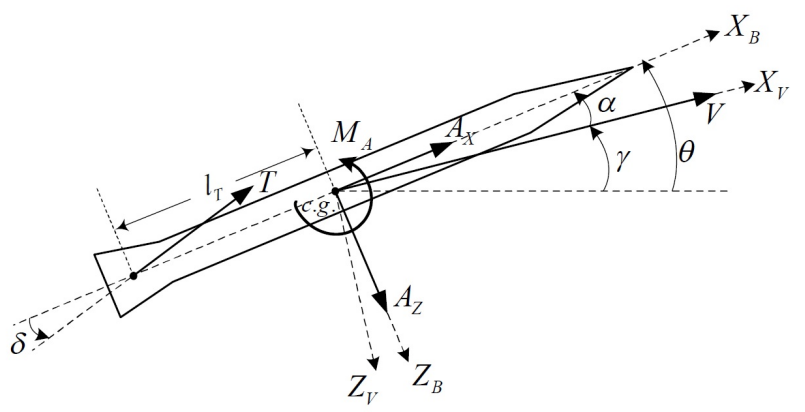

Fig. 1. The interceptor geometry and the parameter definitions

systems $\left(X_{B}-Z_{B}\right)$ and $\left(X_{V}-Z_{V}\right)$ represent the body frame and the velocity frame, respectively. The parameters $\alpha, \theta$, and $\gamma$ denote the angle-of-attack (AOA), the pitch attitude angle, and the flight path angle (FPA), respectively. The parameters $T$ represents the thrust and $\delta$ is the deflection angle of the thrust vector. The parameter denoted by $l_{T}$ represents the length between the center of gravity and the center of thrust (i.e., the position where the thrust acts). The interceptor speed is denoted by $V$. The parameters $A_{X}, A_{Z}$, and $M_{A}$ are the aerodynamic forces and moment given as:

$$
A_{X}=Q S C_{X}, \quad A_{Z}=Q S C_{Z}, \quad M_{A}=Q S d C_{M}
$$

where $C_{X}, C_{Z}$, and $C_{M}$ represent the aerodynamic coefficients. The variables $Q, S$, and $d$ are dynamic pressure, reference area, and reference length, respectively. These aerodynamic forces and moment exist only in the endoatmosphere. Let $a_{X}^{B}$ and $a_{Z}^{B}$ be the body accelerations along $X$ - and $Z$-axis. These accelerations can be expressed as

$$
a_{X}^{B}=\frac{Q S C_{X}+T \cos \delta}{m}, \quad a_{Z}^{B}=\frac{Q S C_{Z}-T \sin \delta}{m}
$$

Here, the variable $m$ is the mass. The acceleration of the velocity frame can also be expressed as:

$$
\left[\begin{array}{l}
a_{X}^{V} \\
a_{Z}^{V}
\end{array}\right]=\left[\begin{array}{cc}
\cos \alpha & \sin \alpha \\
-\sin \alpha & \cos \alpha
\end{array}\right]\left[\begin{array}{c}
a_{X}^{B} \\
a_{Z}^{B}
\end{array}\right]
$$

Then, the equation of motion in the pitch plane can be written as follows.

$$
\begin{gathered}
\dot{\alpha}=\frac{a_{Z}^{V}}{V}+q \\
\dot{q}=\frac{Q S d C_{M}}{I_{y y}}-\frac{l_{T} T \sin \delta}{I_{y y}}
\end{gathered}
$$

where $q$ and $I_{y y}$ are the body pitch rate and the moment of inertia, respectively.

\section{B. Control Strategy}

To configure a single controller applicable to both endoand exo-atmospheres, the acceleration perpendicular to the velocity vector (i.e., $a_{Z}^{V}$ ) is selected as the output variable to be controlled. For convenience, this paper calls this normal acceleration. This control strategy is also good for FPA control because $a_{Z}^{V}$ can change the velocity vector directly. During flight from the endo-atmosphere, the normal acceleration is induced by both $a_{X}^{B}$ and $a_{Z}^{B}$ as

$$
a_{Z}^{V}=-a_{X}^{B} \sin \alpha+a_{Z}^{B} \cos \alpha
$$

In the exo-atmosphere, the normal acceleration is induced only by $a_{X}^{B}$ as

$$
a_{Z}^{V}=-a_{X}^{B} \sin \alpha
$$

By taking the time derivative of $a_{Z}^{V}$ from Eq. (6), we can determine the rate of normal acceleration as

$$
\dot{a}_{Z}^{V}=\eta \dot{\alpha}
$$

where $\eta=\eta_{A}+\eta_{T}$, which are given by

$$
\begin{aligned}
& \begin{aligned}
\eta_{A}= & \frac{Q S C_{Z, \alpha} \cos \alpha}{m}-\frac{Q S C_{Z} \sin \alpha}{m}-\frac{Q S C_{X, \alpha} \sin \alpha}{m} \\
& -\frac{Q S C_{X} \cos \alpha}{m}
\end{aligned} \\
& \eta_{T}=\frac{T \sin \delta \sin \alpha}{m}-\frac{T \cos \delta \cos \alpha}{m}
\end{aligned}
$$

where $C_{X, \alpha}=\partial C_{X} / \partial \alpha$ and $C_{Z, \alpha}=\partial C_{Z} / \partial \alpha$. As shown in Eq. (8), the rate of normal acceleration is directly proportional to the rate of AOA. The parameters $\eta_{A}$ and $\eta_{T}$ can be considered as the effectiveness of aerodynamic force and thrust respectively when generating the normal acceleration. The parameter $\eta$ represents the total effectiveness. Under small angle assumptions of $\alpha$ and $\delta$, these parameters can be approximated by ignoring the non-dominant terms as follows.

$$
\eta_{A} \approx \frac{Q S C_{Z, \alpha}}{m}, \quad \eta_{T} \approx-\frac{T}{m}
$$

From Eq. (11), because of $C_{Z, \alpha}<0$, both $\eta_{A}$ and $\eta_{T}$ have the same sign. Note that the proportional relationship between the rate of normal acceleration and the rate of AOA is maintained for both endo- and exo-atmospheres. This means that we can design a single control structure that can be used in both environments without changing the control structure configuration. Only the magnitude of the proportional factor (i.e., effectiveness) varies from endo- to exo-atmosphere as

$$
\eta=\eta_{A}+\eta_{T} \quad \rightarrow \quad \eta=\eta_{T}
$$

Note that this variation is smooth as dynamic pressure decreases. When considering this variation at the design stage, we can design a controller that can automatically adjusts for environmental changes and effectiveness of aerodynamic and thrust. 


\section{Proposed Controller for AcCeleration CONTROL}

This section describes the autopilot design for normal acceleration control using the feedback linearization technique and the two time-scale separation, via three-loop topology [47]. In the feedback linearization techniques, the choice of the desired error dynamics can determine the control structure. In this paper, we choose the specific desired error dynamics so that the resulting control structure becomes the well-known three-loop control structure.

\section{A. Derivation of New System Equation}

Since $a_{Z}^{V}$ is the output variable to be controlled, we will reconstruct the system equations using the output dynamics. Substituting Eq. (4) into Eq. (8) gives

$$
\dot{a}_{Z}^{V}=\eta\left(\frac{a_{Z}^{V}}{V}+q\right)
$$

From Eq. (5), we assume that $\delta$ is small, then the body pitch rate dynamics is approximated as

$$
\dot{q}=\frac{Q S d C_{M}}{I_{y y}}-\frac{l_{T} T \delta}{I_{y y}}
$$

The, combining Eqs. (13) and (14) provides a new system equation. Here, the state variables are the normal acceleration and the body pitch rate, and the control input is the deflection angle of the thrust vector.

\section{B. Outer Loop Design}

In general, the body pitch rate dynamics is much faster than the normal acceleration dynamics because it is directly affected by the control input. Based on two time-scale separation, the inner and outer loop dynamics can be separated when designing the controller. In the outer loop dynamics, the output variable and the control input can be considered as the normal acceleration and the body pitch rate, respectively. The purpose of the outer loop controller is to generate the body pitch rate command to track the normal acceleration to the desired value. Here we design the desired error dynamics to obtain the first order response of normal acceleration as follows.

$$
\dot{a}_{Z}^{V}-(1 / \tau)\left(a_{c}-a_{Z}^{V}\right)=0
$$

where $a_{c}$ is the acceleration command and $\tau$ is the time constant of the first order response. This is the design parameter of the outer loop controller. Then, applying the feedback linearization method using Eqs. (13) and (15) gives the body pitch rate command as

$$
q_{c}=\frac{1}{\tau \eta}\left(a_{c}-a_{Z}^{V}\right)-\frac{1}{V} a_{Z}^{V}
$$

\section{Inner Loop Design}

Next, the inner loop controller should be designed to track the body pitch rate to the desired value. To this end, let us define the desired error dynamics in a similar way. Here, to achieve the second order response of the body pitch rate, we design the desired error dynamics as

$$
\dot{q}+2 \zeta \omega q+\omega^{2} \int\left(q-q_{c}\right) d t=0
$$

where $q_{c}$ is the body pitch rate command obtained from the outer loop controller. $\zeta$ and $\omega$ represent the damping ratio and the natural frequency which are the design parameters of the inner loop controller. Then, by applying the feedback linearization method using Eqs. (14) and (17), the commanded deflection angle of the thrust vector is obtained as

$$
\delta_{c}=\frac{Q S d C_{M}}{l_{T} T}-\frac{I_{y y}}{l_{T} T} 2 \zeta \omega\left[\frac{\omega}{2 \zeta} \int\left(q_{c}-q\right) d t-q\right]
$$

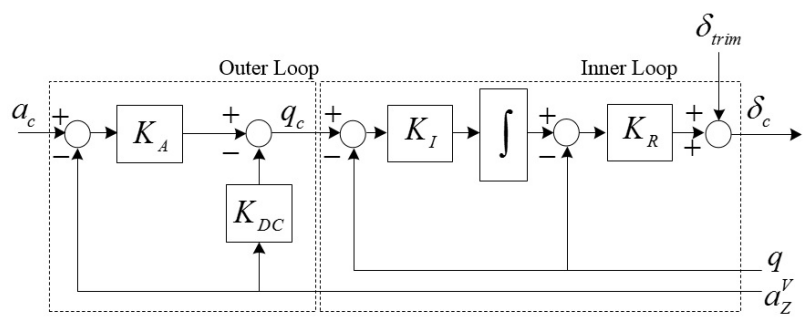

Fig. 2. The overall structure of the proposed controller

\section{Discussion of Controller}

This section discusses the characteristics of the proposed controller. In the outer loop controller, let us define $K_{A}$ and $K_{D C}$ as follows

$$
K_{A}=\frac{1}{\tau \eta}, \quad K_{D C}=\frac{1}{V}
$$

In Eq. (19), these parameters can be regarded as the effective control gain of the outer loop controller. $K_{A}$ is a parametervarying proportional gain, which is given by a function of design parameters $\tau$ and the total effectiveness $\eta$. As $\tau$ and $\eta$ decrease, the value of this gain increases. For a fixed $\tau$, the magnitude of $K_{A}$ in the exo-atmosphere is greater than one in the endo-atmosphere. $K_{A}$ is also automatically adjusted as the flight environment changes. $K_{D C}$ acts as DC gain to make a steady state error of normal acceleration zero.

In a similar way, the effective control gains of the inner loop controller are defined as:

$$
K_{I}=\frac{\omega}{2 \zeta}, \quad K_{R}=-\frac{I_{y y}}{l_{T} T} 2 \zeta \omega, \quad \delta_{t r i m}=\frac{Q S d C_{M}}{l_{T} T}
$$

where $K_{I}$ is an integral gain of the body pitch rate error, which is given by a function of $\omega$ and $\zeta . K_{R}$ is a parametervarying proportional gain which is given by the function of the thrust. $\delta_{\text {trim }}$ can be regarded as the trim command in order to nullify the aerodynamic moment. Accordingly, the trim command becomes zero in the exo-atmosphere.

Fig. 2 represents the overall control structure of the proposed controller. This is similar to the well-known three-loop structure [4-7]. The differences are that $\delta_{\text {trim }}$ is added up to 
the inner loop controller and the effective control gains are automatically scheduled as the operating conditions change.

By applying the inner loop controller designed, we can achieve the second order response as follows.

$$
\frac{q}{q_{c}}=\frac{\omega^{2}}{s^{2}+2 \zeta \omega s+\omega^{2}}
$$

The design parameter $\zeta$ is usually between 0.5 to 1.0 . As $\omega$ increases, fast response of the inner loop is achieved. This value is chosen so that the inner loop response is slower than the actuator response. The rule of thumbs is $\omega<(1 / 3) \omega_{\text {act }}$, where $\omega_{a c t}$ is the natural frequency of the actuator.

Under the outer loop controller designed, the first order response can be achieved as

$$
\frac{a_{Z}^{V}}{a_{c}}=\frac{1}{\tau s+1}
$$

Here, the design parameter $\tau$ decreases, fast response of the outer loop is achieved. This value is chosen so that the achieved outer loop response is slower than the inner loop response.

\section{Simulation Results}

In this section, the numerical simulations are performed to investigate the performance of the proposed controller, by using a nonlinear interceptor model [8]. In this simulation, the actuator system of TVC is modeled as a second order system as follows.

$$
\frac{\delta}{\delta_{c}}=\frac{\omega_{a c t}^{2}}{s^{2}+2 \zeta_{a c t} \omega_{a c t} s+\omega_{a c t}^{2}}
$$

where $\zeta_{a c t}$ and $\omega_{a c t}$ are the damping ratio and the natural frequency of the actuator. In this simulation, the proposed controller is tested under various altitude operating conditions from endo- to exo-atmosphere.

Fig. 3 shows the normal acceleration response normalized by the command for various altitude conditions. As shown in Fig. 3, without changing the controller configuration, we can observe that the proposed controller can provide a uniform response with a significant change in altitude operating conditions. Fig. 4 shows the total effectiveness of aerodynamic force and thrust. This factor is normalized by the effectiveness of thrust. In the endo-atmosphere, the effectiveness of aerodynamic force is about 20 times greater than the effectiveness of thrust. The total effectiveness normalized by the effectiveness of thrust converges to 1 as altitude increases due to lack of aerodynamics in the exoatmosphere. Since the total effectiveness is large in the endo-atmosphere, a small AOA can produce a large normal acceleration. Accordingly, Fig. 5 shows AOA response. As altitude decreases, the magnitude of AOA decreases in steady state. This means that even small AOA can generate enough normal acceleration due to large aerodynamic force. Conversely, in the exo-atmosphere, a relatively small, limited thrust requires a large $\mathrm{AOA}$ to be generated to produce the same amount of normal acceleration as that produced in the endo-atmosphere. Finally, Fig. 6 shows the deflection angle of the thrust vector. Compared to the exo-atmosphere, more deflection angles are needed in the endo-atmosphere in order to nullify aerodynamic moment. This additional deflection angle in the endo-atmosphere can be seen as $\delta_{\text {trim }}$.

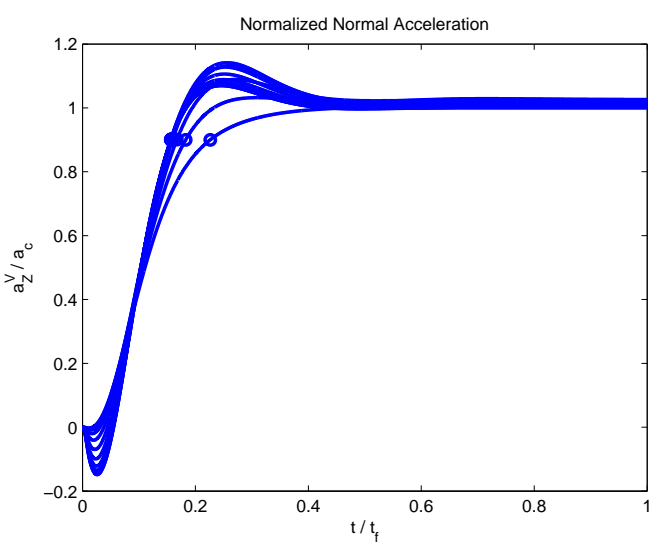

Fig. 3. Normal acceleration normalized by the command

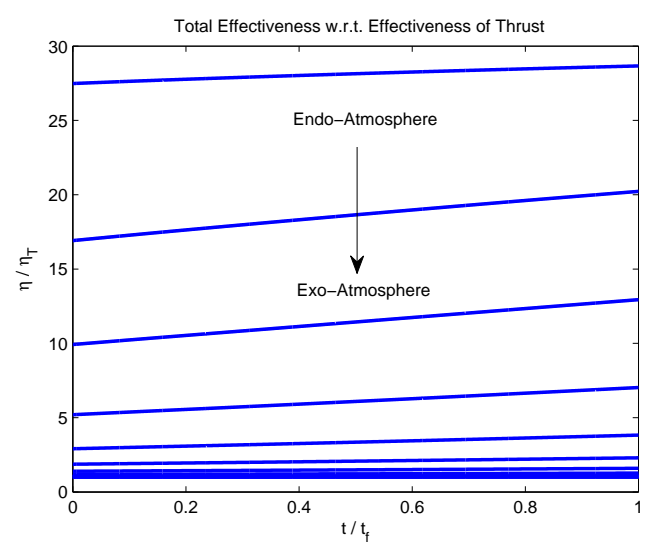

Fig. 4. The total effectiveness with respect to the effectiveness of thrust

\section{CONCLUSIONS}

In this paper, we propose a nonlinear autopilot system that can be used for both endo- and exo-atmospheric interceptors controlled by thrust vector control (TVC) without changing the control configuration. The conventional control strategy of TVC, such as the attitude control, has been challenging when applied to both endo- and exo-atmospheres. Also, in the existing method, the controller structure is required to be changed according to the altitude condition in order to satisfy both environmental conditions. In this paper, by selecting the normal acceleration as an output parameter to be controlled, it is possible to use the same control strategy in both environmental conditions. Based on the assumption of two time scale separations, the feedback linearization technique is used to devise the proposed controller. The desired error dynamics for the inner and outer loops are respectively selected as the first order system and the second order system so that the resulting controller has the well-known three loop control 


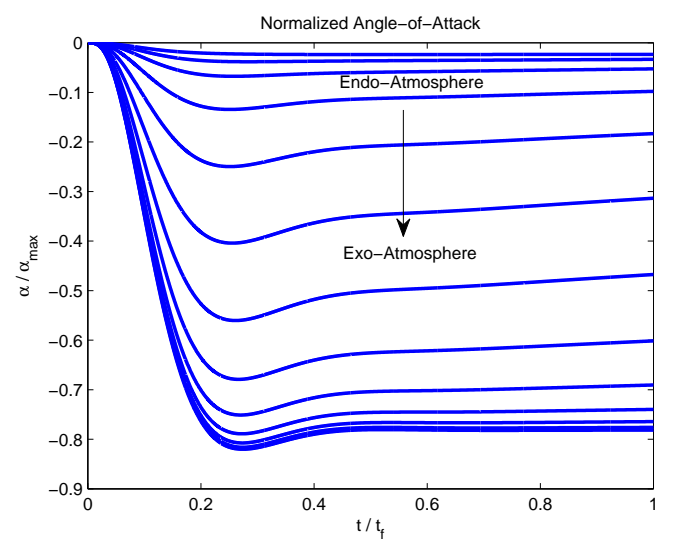

Fig. 5. AOAs normalized by the maximum allowable AOA

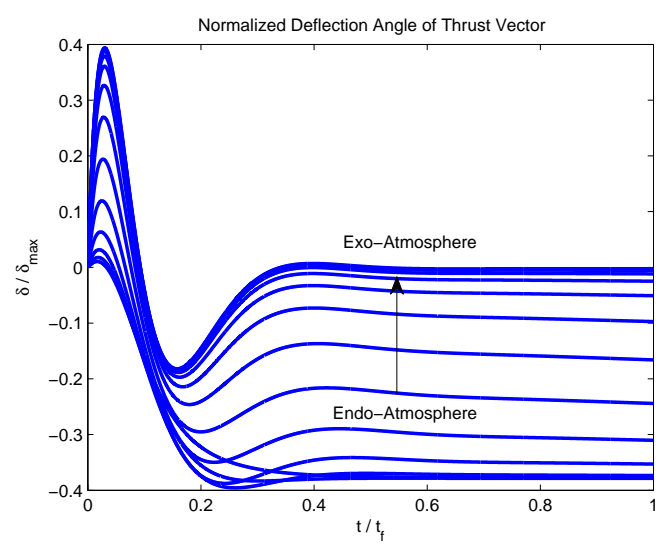

Fig. 6. The deflection angles normalized by the maximum allowable deflection angle

structure with parameter-varying control gains. These gains are automatically scheduled as to the operating conditions change as well as the effectiveness of aerodynamic force and thrust. Therefore, the proposed controller can adaptively allocate aerodynamic force and thrust when generating the required normal acceleration. Aerodynamic force and thrust contribute to produce the normal acceleration in the endoatmosphere. In the exo-atmosphere, the normal acceleration is generated only by thrust. Since the proposed controller is given in a similar form to the well-known control structure, the physical meaning of the proposed controller and the control gains are explicitly presented. Therefore, we can have confidence in the reliability of the proposed controller when implemented in a real system. Finally, the simulation results provide the validity of the proposed method.

\section{REFERENCES}

[1] A. Lichtsinder, E. Kreindler, B. Gal-Or, Minimum-time maneuvers of thrust-vector aircraft, Journal of Guidance, Control, and Dynamics, Vol. 21, No. 2, pp. 244-250, 1998.

[2] D. B. Spencer, Designing continuous-thrust low-earth-orbit to geosynchronous-earth-orbit transfers, Journal of Spacecraft and Rockets, Vol. 32, No. 6, pp. 1033-1038, 1995.

[3] F. K. Yeh, H. H. Chien, and L. C. Fu, Design of Optimal Midcourse Guidance Sliding-Mode Control for Missiles with TVC, IEEE Trans- actions on Aerospace and Electronic Systems, Vol. 39, No. 3, pp. 824-837, 2003.

[4] E. Devaud, J. P. Harcaut, and H. Siguerdidjane, Three-Axes Missile Autopilot Design: From Linear to Nonlinear Control Strategies, Journal of Guidance and Control, and Dynamics, Vol. 24, No. 1, pp. 64-71, 2001.

[5] C. H. Lee, B. E. Jun, J. I. Lee, and M. J. Tahk, Nonlinear Missile Autopilot Design via Three Loop Topology and Time-Delay Adaptation Scheme, 13th International Conference on Control, Automation and Systems (ICCAS), Gwangju, Korea, Oct. 2013.

[6] C. H. Lee, B. E. Jun, and J. I. Lee, Connections between Linear and Nonlinear Autopilots via Three-Loop Topology, Journal of Guidance, Control, and Dynamics, Vol. 39, No. 6, pp. 1426-1432, 2016.

[7] R. Padhi, C. Sirisha, A. K. Sarkar, P. K. Kar, T. Srinivasan, Nonlinear and Linear Autopilot Performance Comparison of Tactical Flight Vehicle, AIAA Guidance, Navigation, and Control Conference, 2012.

[8] P. H. Zipfel, Modeling and Simulation of Aerospace Vehicle Dynamics 2nd edition, AIAA, Inc. 1801 Alexander Bell Drive Reston, VA 201914344, 2007. 
2017-07-20

Nonlinear acceleration controller for exo-atmospheric and endo-atmospheric interceptors with TVC

\author{
Lee, Chang-Hun
}

IEEE

Lee $\mathrm{CH}$, Jun BE, Shin HS, Tsourdos A. (2017) Nonlinear acceleration controller for exo-atmospheric and endo-atmospheric interceptors with TVC. IEEE 25th Mediterranean

Conference on Control and Automation (MED), 3-6 July 2017, Valletta, Malta

htp://dx.doi.org/10.1109/MED.2017.7984110

Downloaded from Cranfield Library Services E-Repository 